\title{
Impact of High-Altitude Hypoxia on Early Osseointegration With Bioactive Titanium
}

\author{
Yarong Wang ${ }^{1}$, Zekun Gan ${ }^{1}$, Haibin Lü ${ }^{2}$ Ziyi Liu ${ }^{1}$, Peng Shang ${ }^{3}$, Jian Zhang ${ }^{3}$, Wuwei Yin ${ }^{1}$, \\ Hongxing Chu ${ }^{1}$, Renlei Yuan ${ }^{4}$, Yingxin Ye ${ }^{1}$, Pei Chen $^{1 *}$ and Mingdeng Rong ${ }^{1 *}$ \\ 'Department of Periodontology and Implantology, Stomatological Hospital, Southern Medical University, Guangzhou, China, \\ ${ }^{2}$ Department of Implantology, Stomatological Hospital, Southern Medical University, Guangzhou, China, ${ }^{3}$ College of Animal \\ Science, Tibet Agriculture and Animal Husbandry University, Linzhi, China, ' ${ }^{4}$ inzhi People's Hospital, Linzhi, China
}

Nowadays, the bone osseointegration in different environments is comparable, but the mechanism is unclear. This study aimed to investigate the osseointegration of different bioactive titanium surfaces under normoxic or high-altitude hypoxic environments. Titanium implants were subjected to one of two surface treatments: (1) sanding, blasting, and acid etching to obtain a rough surface, or (2) extensive polishing to obtain a smooth surface. Changes in the morphology, proliferation, and protein expression of osteoblasts on the rough and smooth surfaces were examined, and bone formation was studied through western blotting and animal-based experiments. Our findings found that a hypoxic environment and rough titanium implant surface promoted the osteogenic differentiation of osteoblasts and activated the JAK1/STAT1/HIF-1 $\alpha$ pathway in vitro. The animal study revealed that following implant insertion in tibia of rabbit, bone repair at high altitudes was slower than that at low altitudes (i.e., in plains) after 2 weeks; however, bone formation did not differ significantly after 4 weeks. The results of our study showed that: (1) The altitude hypoxia environment would affect the early osseointegration of titanium implants while titanium implants with rough surfaces can mitigate the effects of this hypoxic environment on osseointegration, (2) the mechanism may be related to the activation of JAK1/STAT1/ $\mathrm{HIF-1} \alpha$ pathway, and (3) our results suggest the osteogenesis of titanium implants, such as oral implants, is closely related to the oxygen environment. Clinical doctors, especially dentists, should pay attention to the influence of hypoxia on early osseointegration in patients with high altitude. For example, it is better to choose an implant system with rough implant surface in the oral cavity of patients with tooth loss at high altitude.

Keywords: osseointegration, bioactive titanium, hypoxia, normoxia, osteoblast

\section{INTRODUCTION}

The theory of "osseointegration" has existed for more than 50 years (Lee and Bance, 2019). Several basic and clinical studies have confirmed that formation of the osseointegration interface of dental implants is the basis for successful implant restoration (Chen et al., 2019; Lee and Bance, 2019). Although the current success rate of implant restoration in 
plains and flatlands is relatively high, approximately $1-2 \%$ of patients experience implant failure due to the lack of binding at the initial stage of implant insertion (Guglielmotti et al., 2019). Meanwhile, the hypoxic atmosphere characteristic of high-altitude environments in plateau regions is associated with a higher risk of osteoporosis and a longer healing time of bone fractures in the elderly population compared to those from regions of low altitudes, such as plains (Bernardi et al., 2020; Feng et al., 2020). This suggests that oxygen concentration is an important factor for bone defect repair. However, to date, the success rate of oral planting and relevant clinical guidelines in plateaus remains unreported. Thus, the only available clinical guidelines related to planting are specific to plains regions with constant oxygen (Guglielmotti et al., 2019). In this study, the osteogenesis of implants in different environment was evaluated in Xizang, China (highland zone, altitude near $3,000 \mathrm{~m}$, severe cold; Liu et al., 2021) and Guangzhou, China (plain zone, altitude near 1,000 m, Warm and rainy; Liu et al., 2020a).

Bone defect repair is a complex and multifactorial interactive process (Mavrogenis et al., 2009; Dashnyam et al., 2019). Bone trauma and bone defects lead to local vascular rupture followed by bleeding, which leads to tissue ischemia, hypoxia, and acidosis (Ma et al., 2020; Wu et al., 2020a). This pathological process is not conducive to the growth and repair of bone tissue; thus, the application of tissue engineering to repair and restore bone defects has become a popular and important research topic (Wu et al., 2020b). Current research is focused on establishing a suitable microenvironment for the growth and proliferation of osteoblasts in bone defects and promoting the growth and differentiation of new bone to ultimately form a normal structure with normal function (Bernardi et al., 2020; Feng et al., 2020; Wen and Lv, 2020).

Autogenous bone grafts are typically the first choice of material for use on large bone defects; however, their application is restricted due to limited sources, the risk of infection, and adverse immune responses (Gonzaga et al., 2019; Brunello et al., 2020). Titanium and titanium alloys are widely used in the medical field, particularly in orthopedics due to their stable chemical properties, accessibility, and excellent resistance to corrosion (Lee et al., 2017; Talley et al., 2018). Meanwhile, physical, chemical, and biological surface modification methods can improve the roughness of titanium mesh and promote the adhesion and proliferation of osteoblasts (Lee et al., 2017; Talley et al., 2018). However, research on the effects of modified materials under high-altitude hypoxia is still in the exploratory stages.

In the present study, the microenvironment of bone defects was simulated by establishing a hypoxia model and bone defect model of the rabbit tibial plateau. We aimed to observe and compare osteoblasts cultured on titanium disks with different surfaces under normoxic or hypoxic conditions. The effect of these conditions on the morphology, proliferation, and repair of bone defects has the potential to provide a theoretical basis for the clinical application of titanium plate modification in the treatment of bone defects under normoxic or hypoxic conditions.

\section{MATERIALS AND METHODS}

\section{Titanium Plate Preparation}

Pure industrial TA2 titanium disks (TA2, Baoji, Shanxi Province, China) were customized ( $10 \mathrm{~mm}$ diameter and $2 \mathrm{~mm}$ thickness) and divided into two groups based on their altered surface (smooth and rough) for evaluation. To obtain a smooth surface, the disks were gradually polished using 600\#, 800\#, 1,200\#, and 1,500\# metallographic sandpaper and washed in an ultrasonic bath. To obtain a rough surface, the disks were sandblasted using alumina particles $(90-250 \mu \mathrm{m}$ in diameter, at $4.5 \mathrm{kPa}$ and $90^{\circ} \mathrm{C}$ for $30 \mathrm{~s}$ ) and treated with a mixture of $18 \%$ hydrochloric acid and $49 \%$ sulfuric acid at $60^{\circ} \mathrm{C}$ for $40 \mathrm{~min}$ to complete the acid etching process.

\section{Cell Culture and Establishment of the Hypoxia Model}

MG-63 cells (Sigma, United States) were cultured in minimum Eagle's medium (MEM, Gibco, NY, United States) supplemented with $10 \%$ high-quality fetal bovine serum (FBS, Gibco, United States) and 1\% penicillin-streptomycin antibiotic solution (Beyotime, Beijing, China). The culture medium was replaced every 2-3 days. Once the cells reached $80 \%$ confluence, they were passaged at a ratio of $1: 3$ and incubated with $5 \% \mathrm{CO} 2$ at $37^{\circ} \mathrm{C}$ in a saturated humidity incubator (Sanyo, Toshima-ku, Japan). The MG-63 cell suspension was collected and its concentration adjusted to $1 \times 10^{5}$ cells $/ \mathrm{ml}$. A titanium plate was placed in a 48-well plate (Corning, NY, United States), and $100 \mu \mathrm{l}$ of cell suspension was added to the surface of each disk. The plate was then incubated for $3 \mathrm{~h}$, and cell adherence to titanium was confirmed by scanning electron microscopy (SEM). Following cell adherence, $400 \mu \mathrm{l}$ of culture medium was added to each well. The 48 -well plate was then transferred into a portable hypoxia cell culture device (Billups-Rothenberg, NY, United States) and continuously supplied with mixed gas $\left(90 \% \mathrm{~N}_{2}, 5 \% \mathrm{CO}_{2}\right.$, and $\left.5 \% \mathrm{O}_{2}\right)$. The hypoxia device was placed in an incubator at $37^{\circ} \mathrm{C}$ to maintain saturated humidity levels. In osteogenic condition, the osteogenic induction medium was added (MEM with $10 \% \mathrm{FBS}, 10 \mathrm{mmol} / \mathrm{L}, \beta$-glycerophosphate, $10 \mathrm{nmol} / \mathrm{L}$ dexamethasone, and $50 \mu \mathrm{g} / \mathrm{mL}$ ascorbic acid).

\section{Cell Proliferation Assay}

MG-63 cell proliferation was determined using the Cell Counting Kit-8 (CCK-8, Sigma, MO, United States) and EdU assay (Sigma). For the CCK-8 assay, the MG-63 cell suspension was adjusted to $1 \times 10^{5}$ cells $/ \mathrm{ml}$ and $100 \mu \mathrm{l}$ was seeded on a titanium plate in a 48-well plate. Following adherence, the cells were cultured under normoxia and hypoxia conditions for 24 and $48 \mathrm{~h}$. When the hypoxia treatment reached the corresponding time point, the CCK- 8 assay was carried out according to the manufacturer's protocol. Briefly, the CCK- 8 solution was added to each well and incubated for $3 \mathrm{~h}$. Absorbance was measured at $450 \mathrm{~nm}$ using a microplate reader.

An EdU assay was performed by treating $5 \times 10^{3}$ MG-63 cells with $50 \mathrm{mM} \mathrm{EdU}$ at $37^{\circ} \mathrm{C}$ for $6 \mathrm{~h}$. The cells were stilled attached to the titanium in this assay. After fixation in $4 \%$ 
paraformaldehyde, MG-63 cells were treated with glycine for $10 \mathrm{~min}$ and $0.5 \%$ Triton X-100 for $10 \mathrm{~min}$. The cells were then mixed with 4,6-diamidino-2-phenylindole (DAPI) in the dark for $30 \mathrm{~min}$, and images were captured by fluorescence microscopy.

\section{Morphological Assessment of the Different Bioactive Titanium Surfaces}

Scanning electron microscopy was employed to observe the titanium disk morphologies. MG-63 cells were inoculated on both smooth and rough disks and cultured for $24 \mathrm{~h}$, followed by fixing with pre-cooled $3 \%$ glutaraldehyde overnight at $4^{\circ} \mathrm{C}$. Next, each sample was fixed with pre-cooled $1 \%$ acetic acid at $4^{\circ} \mathrm{C}$ for $1 \mathrm{~h}$. The samples were then dehydrated for $10 \mathrm{~min}$ using acetone/isoamyl acetate $(1: 1)$ and dehydrated for $30 \mathrm{~min}$

TABLE 1 | Gene sequences.

\begin{tabular}{ll}
\hline Gene & Sequence (5'-3') \\
\hline BMP2 & ACCCGCTGTCTTCTAGCGTITTCAGGCCGAACATGCTGAG \\
COL1 & GAGGGCCAAGACGAAGACATCCAGATCACGTCATCGCACAAC \\
RUNX2 & TGGTTACTGTCATGGCGGGTATCTCAGATCGTTGAACCTTGCTA \\
GAPDH & GGAGCGAGATCCCTCCAAAATGGCTGTTTCATACTTCTCATGG
\end{tabular}

with isoamyl acetate. Each dehydrated sample was immersed first in a 520\% acetonitrile solution, followed by $15-\mathrm{min}$ immersions in $70,80,90,95$, and $100 \%$ acetonitrile solutions, and a final transfer to a solution of $100 \%$ acetonitrile. The samples were then vacuum-dried for $30 \mathrm{~min}$ and coated with carbon and gold particles for observation under SEM.

\section{Western Blot Analysis}

MG-63 cells cultured on the different bioactive titanium disks under normoxia and hypoxia were collected and fully lysed using RIPA Lysis Buffer (Beyotime, China). Protein content was quantified using a bicinchoninic acid kit (Beyotime, China). After adjusting the sample concentrations, SDS-polyacrylamide gel electrophoresis (Amresco, OH, United States) was carried out using $30 \mu \mathrm{g}$ of protein per sample. The separated protein bands were then transferred to a nitrocellulose membrane and incubated in a blocking buffer solution containing 5\% skimmed milk powder (Sangon Biotech, China) overnight at $4^{\circ} \mathrm{C}$. Next, the membrane was washed with TBST, incubated with goat anti-human p-JAK1, p-STAT1, JAK1, STAT1, HIF-1 $\alpha$, BMP2, COL-1, and RUNX2 antibodies (Abcam, 1:1000, United States), and visualized using ECL reagent (Pierce, IL, United States). Membranes were cleared with a clearing buffer and re-incubated

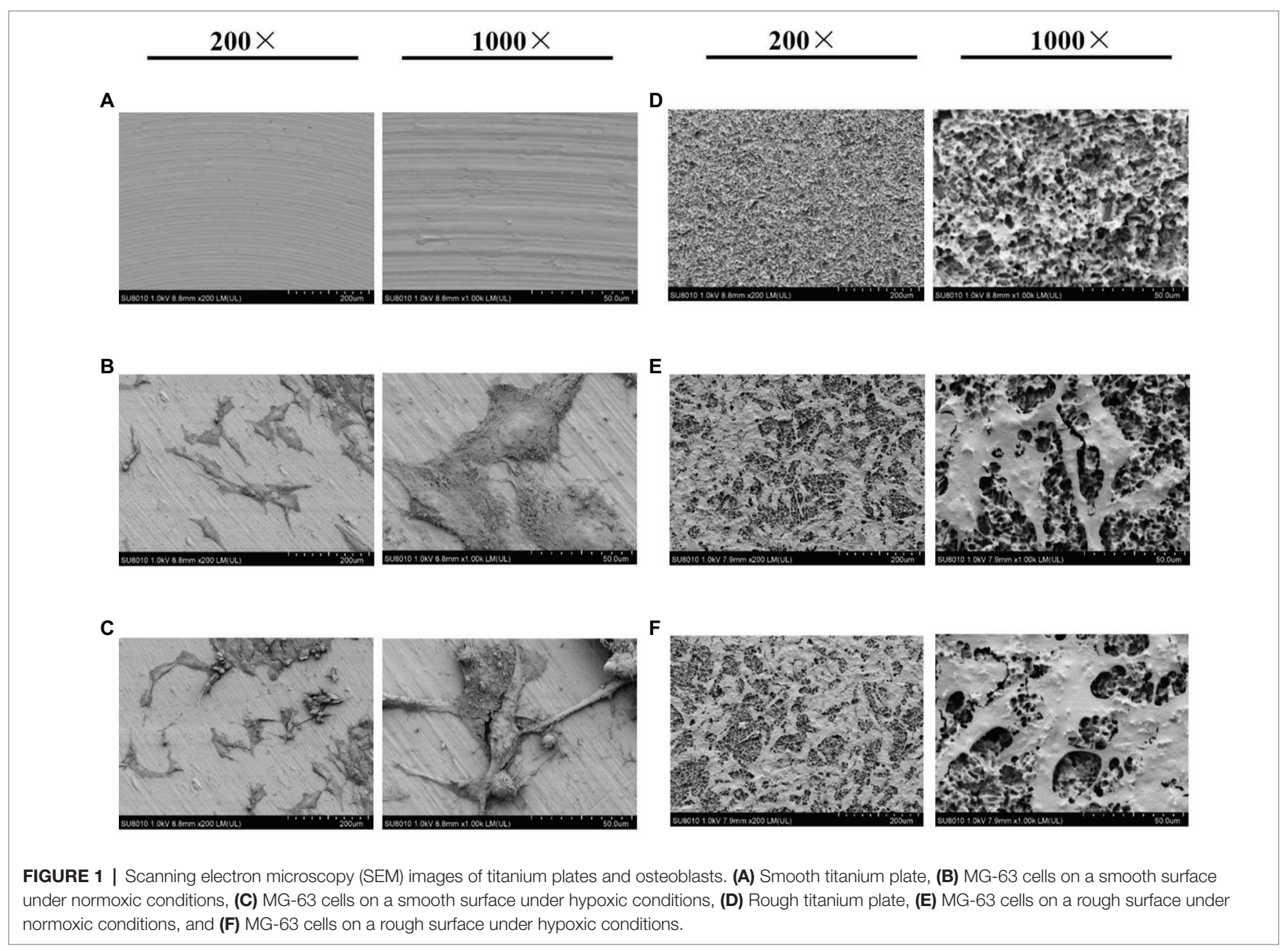


with GAPDH (Abcam, 1:5,000, United States) for visualization as an internal reference. The results were analyzed using the Chemi-Genius gel imaging system for the expression of the target protein.

\section{RT-qPCR}

RNA was extracted using TRIzol and reverse-transcribed into cDNA using a reverse transcription kit (Tiangen, China) according to the manufacturer's instructions. Next, the RNA content was detected using fluorescence (Bio-Rad, United States). The sequences of the RT-PCR primers used are listed in Table 1. The reaction cycle was carried out at $95^{\circ} \mathrm{C}$ for $20 \mathrm{~s}$, annealing at $60^{\circ} \mathrm{C}$ for $30 \mathrm{~s}$, and extension at $72^{\circ} \mathrm{C}$ for $30 \mathrm{~s}$. The results were calculated using the $2^{-\Delta \Delta \mathrm{CT}}$ method.

\section{Animal Experiments}

The protocol for our animal experiments was approved by the Ethics Committee of Long Gui Xing Ke Animal Farm, Baiyun District, Guangzhou. Titanium implants from different groups $(3.45 \mathrm{~mm} \times 10 \mathrm{~mm})$ were implanted into four adult male New Zealand white rabbits (aged 20 weeks and weighing approximately $3.0 \mathrm{~kg}$ ). Animal experiments were performed in a plain zone (Guangzhou, China) and a highland zone (Xizang, China). Before experiments, all animals were lived in the correspondent place at least 6 months in the SPF environment. Animals were anesthetized using Sumian Xin (Animal Husbandry Research Institute, Jilin, China) at $0.15 \mathrm{ml} /$ $\mathrm{kg}$ body weight. Preoperatively, $0.6 \mathrm{ml}$ of Primacaine (Merignac Cedex, France; $0.2 \mathrm{ml} / \mathrm{kg}$ of body weight) was locally injected into the surgical site of the tibia. The titanium plates were implanted approximately $7 \mathrm{~mm}-12 \mathrm{~mm}$ beneath the joint, and a similar operation was performed on the other side of the tibia. The rough plate was placed in one side, and the smooth plate was placed in other side. After the operation, all animals were injected with $0.3 \mathrm{mg}$ (Pharmaceutical Company, Sichuan, China) and allowed to move freely. At either 2 or 4 weeks post-surgery ( $n=5$ at each time point), the animals were sacrificed by excessive anesthetic $(1.5 \mathrm{ml} / \mathrm{kg})$ and samples were collected for histological analysis. Bone grafts and soft tissues were collected and fixed in 10\% neutral-buffered formalin (Cohen et al., 2020).

\section{Bone-to-Implant Contact Evaluation}

Tissue specimens were dehydrated in a gradient dilution of ethanol, immersed in $100 \%$ resin, and embedded in methyl methacrylate. They were then cut in the buccolingual direction and parallel to the axis of the implants using a low-speed diamond saw (SP1600, Leica Biosystems, Germany). The section containing the implant was ground until its thickness reached 60-80 $\mu \mathrm{m}$. It was then stained with methylene blue-acid fuchsin. Each specimen was observed under an optical microscope (Olympus BX41, Olympus Co., Japan) and analyzed using the OsteoMeasure $^{\mathrm{TM}}$ software. Bone-to-implant contact (BIC) was quantitatively measured as follows: BIC (\%) = (sum of the length of $\mathrm{BIC}) /($ circumference of the implant chamber region $) \times 100$. $\mathrm{BIC}$ was defined as the interface where the bone tissue was located within $20 \mu \mathrm{m}$ of the implant surface without any intervention of the soft tissue.

\section{Statistical Analysis}

Each measurement was repeated in triplicate and averaged. Data were collected and reported as the mean $\pm \mathrm{SD}$. One-way ANOVA was carried out between the compared groups, followed by the Student-Newman-Keuls method. All analyses were conducted using SPSS Statistics 17, and the significance level was set at 0.05 .

\section{RESULTS}

\section{Morphological Characteristics of the Different Titanium Surfaces}

Scanning electron microscopy analysis showed that mechanical polishing resulted in a smooth, "mirror-like" titanium surface (Figure 1A), whereas the sandblasted and acid-etched surface was rough and displayed a "honeycomb" structure with different porosities (Figure 1D). MG-63 cells were inoculated on the two titanium surfaces. Under normoxic or hypoxic conditions, titanium disks with a smooth surface contained numerous cells connected by cell matrix that grew in a single layer and with a flat appearance (Figures 1B,C). Conversely, under normoxic and hypoxic conditions, disks with a rough surface were covered with multiple cell layers connected by the matrix, with fractures at multiple sites, unclear boundaries, and unevenness (Figures 1D-F).

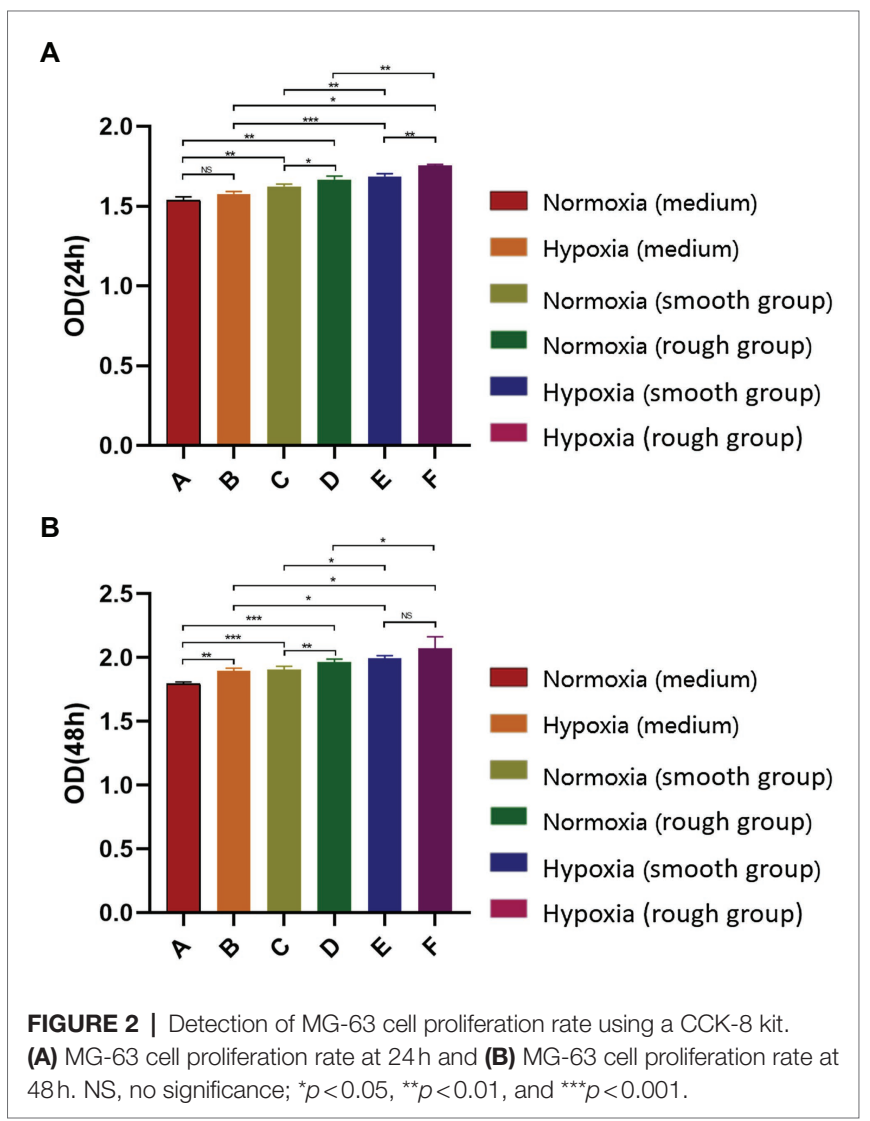




\section{Titanium Plates Promote Cell Proliferation Under Hypoxia}

At both 24 and $48 \mathrm{~h}$, the hypoxic condition was better than the normoxic one in term of proliferation $(p<0.05$; Figures 2A,B). After $48 \mathrm{~h}$, the EdU assay showed that cell proliferation was significantly higher under hypoxia than normoxia. Moreover, cell viability was higher on titanium disks with a rough surface than on those with a smooth surface (Figure 3). Our findings suggest that hypoxic conditions might benefit cell proliferation. Additionally, the two surface treatments improved cell proliferation, with a particularly significant affect induced by the rough surface.

\section{A Rough Surface Enhances Osteogenic Differentiation Under Hypoxia}

To evaluate the osteogenic differentiation of MG-63 cells in environments with different oxygen levels and on different surfaces, the expression of osteogenic-related proteins was detected 14 days after culture. The results showed that the expression of osteogenic-related proteins was promoted under
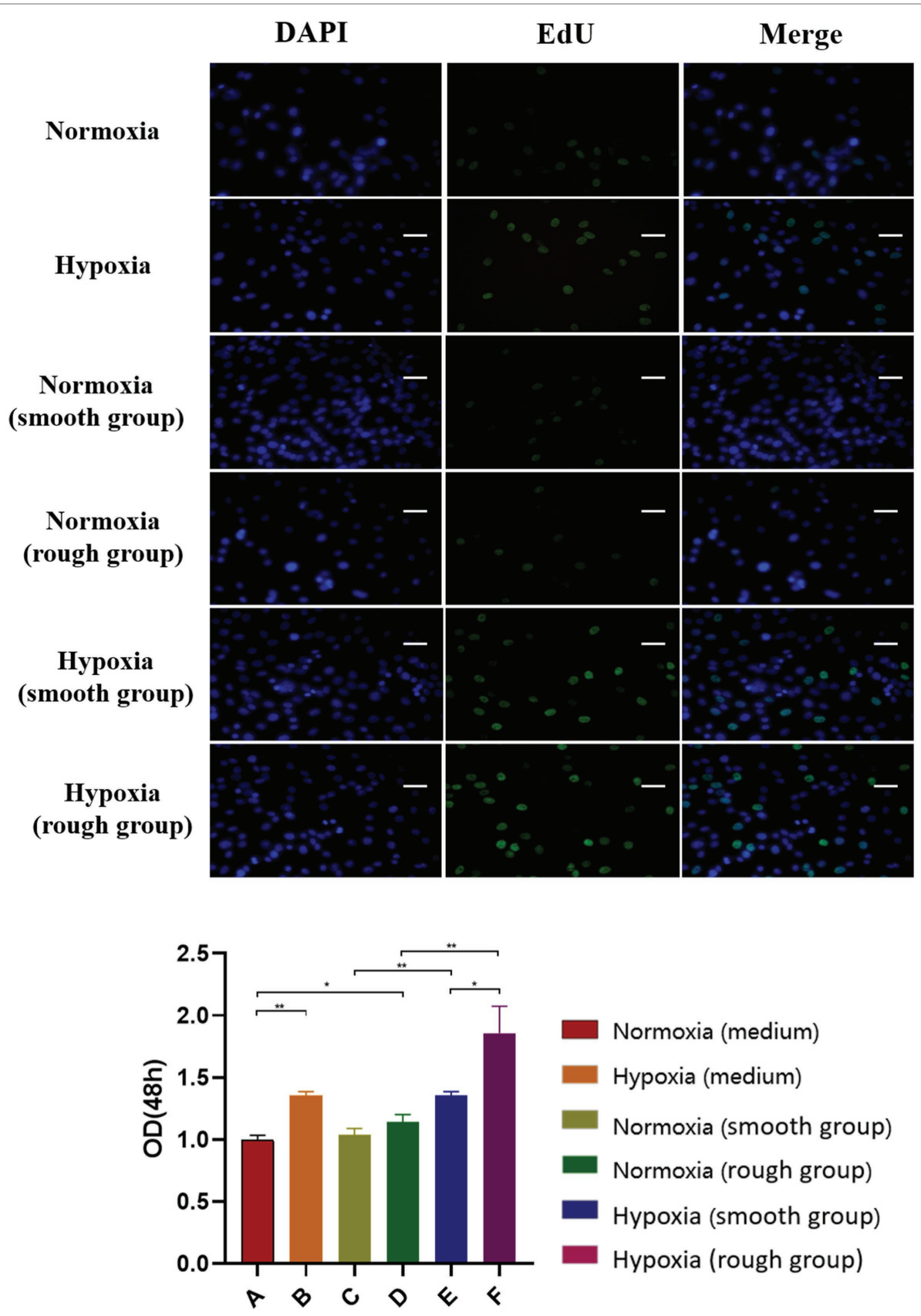

FIGURE 3 | EdU staining results for the different groups. The EdU staining results and quantification of different groups under different environment [Blue: 4,6-diamidino-2-phenylindole (DAPI), Green: EdU]. Scale bar $=200 \mu \mathrm{m} .{ }^{*} p<0.05,{ }^{* *} p<0.01$. 
hypoxic conditions and further enhanced by the rough surface (Figure 4A; BMP2, $p<0.05$; COL-1 $p<0.05$; RUNX2, $p<0.01)$. These results were confirmed by the RT-qPCR findings with the rough surface found to increase the expression of genes associated with osteogenesis in MG-63 cells (Figure 4B).

\section{JAK1/STAT1/HIF-1 $\alpha$ Pathway Activation Under Different Conditions}

From the western blot analysis of MG-63 cells, we determined that the JAK1/STAT1/HIF-1 $\alpha$ pathway displayed a reduced expression (p-JAK1/JAK1, p-STAT1/STAT1, and HIF-1 $\alpha$ / GADPH) under normoxic conditions, but was activated under hypoxic conditions (Figures 5A,B). Although the rough surface promoted activation of the JAK1/STAT1/HIF- $1 \alpha$ pathway (p-JAK1/JAK1, p-STAT1/STAT1, and HIF-1 $\alpha /$ GADPH) under normoxic conditions, no significant differences were observed in the activation of this pathway between the smooth surface and rough surface groups under hypoxia (Figures 5A,B).

\section{Histological Analysis}

Data collected from our animal-based experiments showed that no implant loss occurred at 2 and 4 weeks post-operation. In the high-altitude hypoxic environment, new bone formation was detected on all of the implants, and osseointegration was observed. Compared to implants with a smooth surface, those with a rough surface showed more bone regeneration at 2 weeks post-operation. Intimate bone contact with the implant surface was observed in the healing cavity around the implants; only a small amount of new bone tissue was found on implants with a smooth surface. At 4 weeks in both high-altitude and plain area, new bone covered most of the implants with rough surfaces, whereas relatively few covered areas were detected on implants with smooth surfaces (Figure 6A). By comparing the BIC (\%) of each group, we noted that at week 2, implants

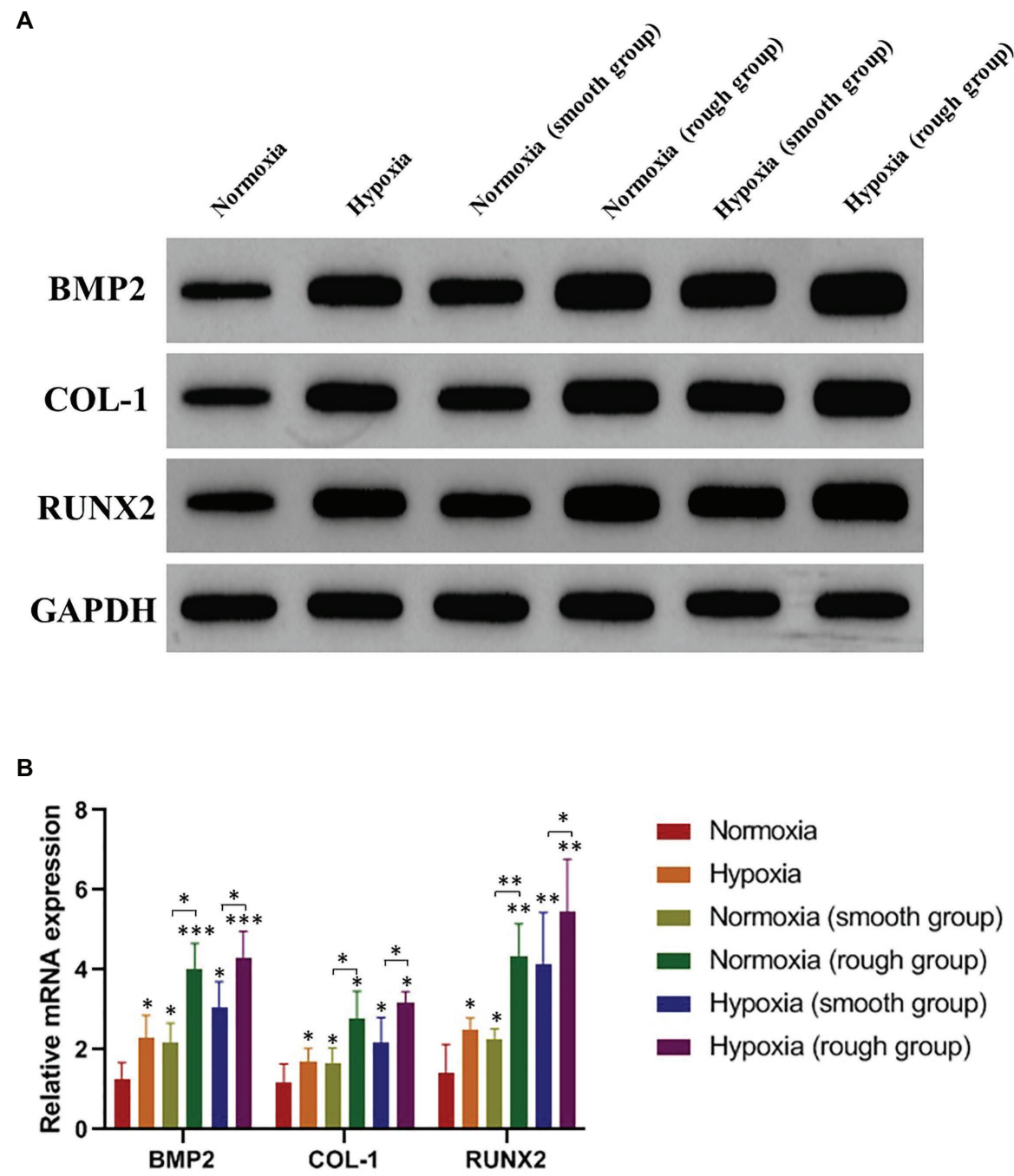

FIGURE 4 | Osteogenic-related proteins and mRNA (BMP2, COL-1, and RUNX2) expression of MG-63 cells in different groups. (A) Western blotting and (B) RT-qPCR. $" p<0.05,{ }^{* *} p<0.01$, and ${ }^{* * *} p<0.001,{ }^{*}$ compared with the corresponding group maintained under normoxic conditions. 
A

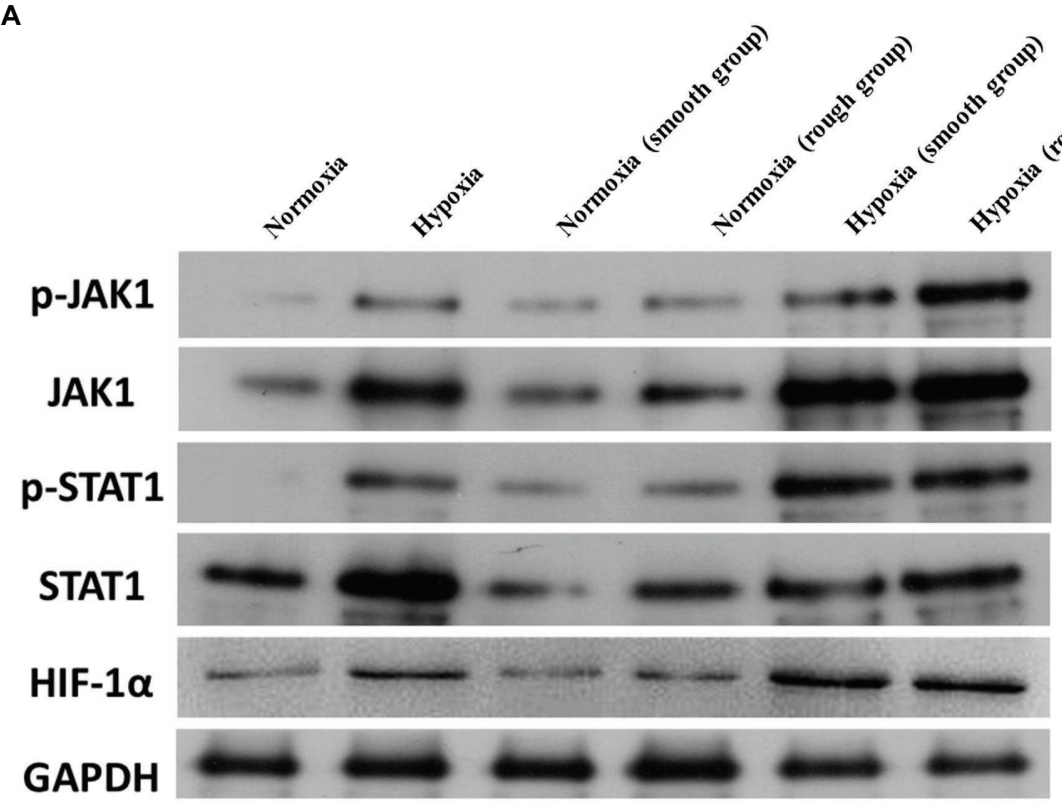

B

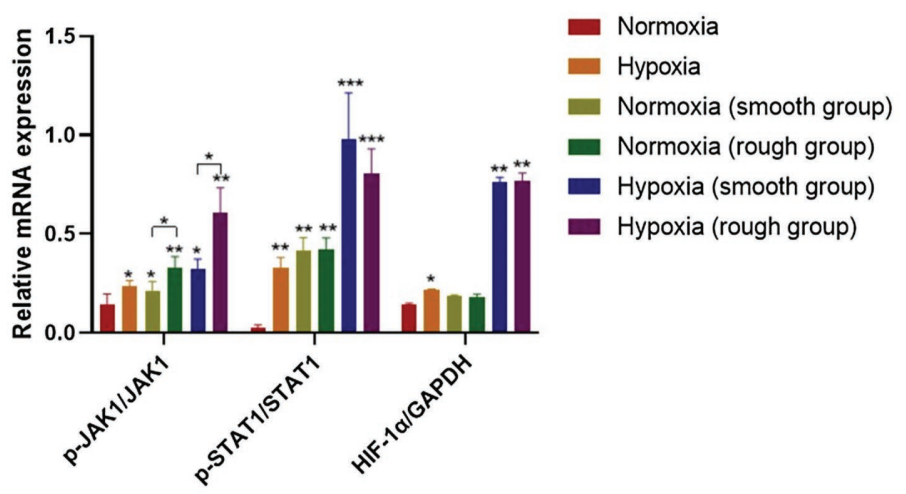

FIGURE 5 | The JAK1/STAT1/HIF-1 $\alpha$ pathway activation in different groups. (A) Protein expression of p-JAK1, JAK1, p-STAT1, STAT1, HIF-1 $\alpha$, and GAPDH; (B) Relative protein expression of $p-J A K 1 / J A K 1$, p-STAT1/STAT1, and HIF-1 $\alpha /$ GAPDH. ${ }^{*} p<0.05$, ${ }^{* *} p<0.01$, and ${ }^{\star \star \star} p<0.001$, ${ }^{\star}$ Compared with the corresponding group maintained under normoxic conditions.

with a rough surface showed superior bone formation and osseointegration capacity compared to those with a smooth surface, under both normoxic and hypoxic conditions (Figure 6B). At week 4, significant differences were detected between smooth and rough surface both in plain and highland. Titanium plates with a rough surface had significantly better osteogenesis to the hypoxic microenvironment compared to those with a smooth surface and were able to regenerate and bind bone in a hypoxic environment.

\section{DISCUSSION}

Titanium and its alloys are metal materials commonly used in the field of biomedicine (He et al., 2020; Tan et al., 2020), more specifically in the treatment of bone defects, as implant devices to replace damaged or defective tissues. Recent research on titanium surface modifications has focused primarily on slurry spray coating, hydroxyapatite coating, micro-arc oxidation, laser irradiation, and anodic oxidation (Liu et al., 2017; He et al., 2019; Pippenger et al., 2019). Moreover, studies have reported that surface treatment of uncoated grafts is superior to that of coated grafts, for example, in terms of outstanding biocompatibility and osteoconductive properties (Horvathy et al., 2016; Wu et al., 2018a). However, bone formation is not only affected by bone materials, but also by $\mathrm{O} 2$ concentration. Indeed, hypoxic environments can promote bone formation and bone healing (Xue et al., 2020; Yu et al., 2020). Therefore, in the present study, we explored the activities of osteoblasts on the surface of different bioactive titanium implants under normoxic and hypoxic conditions.

Currently, sandblasting and acid etching (SLA) is one of the most widely used non-coating surface treatment methods (Corvino et al., 2020; Lu et al., 2020). SLA combines the 
A

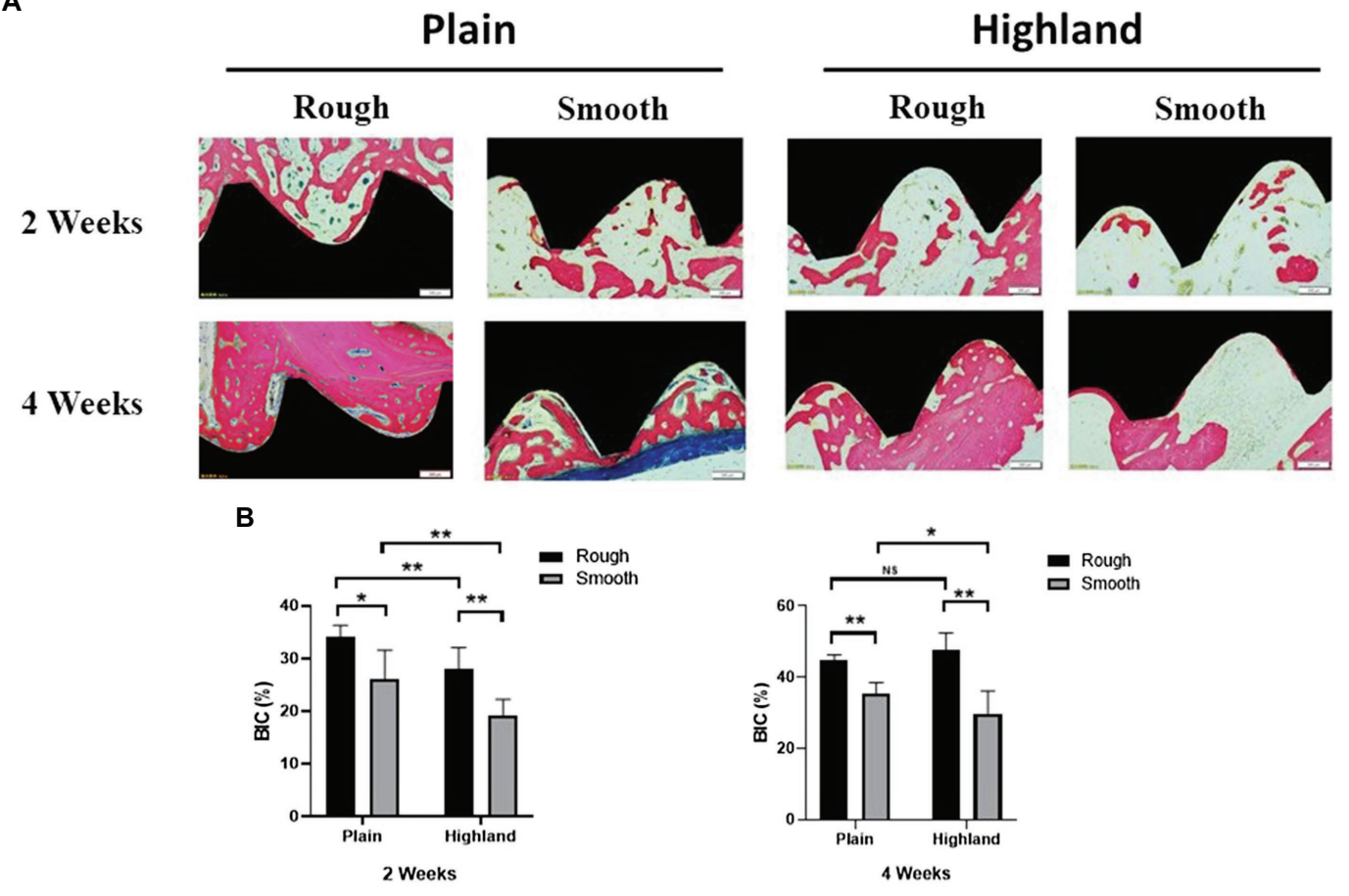

FIGURE 6 | Histological observation of implants and the surrounding tissues [methylene blue-acid fuchsin (A)]; comparison of Bone-to-Implant Contact (BIC; \%) at week 2 and 4 (B). NS, no significance; ${ }^{*} p<0.05,{ }^{* *} p<0.01$.

advantages of physical and chemical etching, while also offering an inexpensive and simple platform (Lu et al., 2020). In our study, irregular honeycomb-shaped holes were formed on the surface of the titanium plate following SLA. Considering that cell adhesion is a prerequisite for the proliferation and differentiation of bone cells, the surface morphology of biomaterials directly affects early adhesion (Kim et al., 2019). Studies have confirmed that SLA not only effectively increases the contact area of osteoblasts, but also enhances their adhesive and proliferative capacity (Bayram et al., 2012; Ding et al., 2015). The results of the CCK- 8 and EdU analyses in the current study support that rough surface promotes cell proliferation under hypoxia. Moreover, at 2 weeks, the expression of osteogenic-related proteins and genes (BMP2, COL-1, and RUNX2) was promoted under hypoxic conditions (Figure 4). The rough surface further enhanced osteogenic differentiation and hypoxia enhanced the expression of JAK1 and STAT1.

The JAK1/STAT1 pathway plays an integral role in the transmission of hypoxia signals and mediates the effects of hypoxia (Yang et al., 2017; Wu et al., 2018b; Liu et al., 2020b). Moreover, mesenchymal stem cells ameliorate hypoxia and reoxygenation injury in renal tubular epithelial cells through the JAK/STAT signaling pathway (Zhang et al., 2017). In addition, activation of the JAK1/STAT1 pathway can promote HIF- $1 \alpha$ expression in hypoxic environments (Yang et al., 2017). Similarly, in our study, activation of the JAK1/STAT1 pathway stimulated HIF-1 $\alpha$ expression by western blotting. Overexpression of HIF- $1 \alpha$ can upregulate the vascular endothelial growth factor, induce neovascularization, and accelerate bone formation ( $\mathrm{Du}$ et al., 2018; Liu et al., 2019). Therefore, hypoxia can enhance osteogenic differentiation via the JAK1/STAT1/HIF- $1 \alpha$ pathway.

Our animal model revealed that bone formation under high-altitude hypoxic conditions was significantly slower than that under normoxia; this result was consistent with previous studies (Lezon et al., 2016; Suresh et al., 2019). In highland environments, the extent of osteogenesis on both rough and smooth surfaces was lower than that observed in plains at 2 weeks. However, no significant difference was observed in the BIC for the rough surface between the two conditions at 4 weeks. Moreover, the roughness of the implant surface was positively associated with the amount of newly generated bone. Studies have reported that high-altitude hypoxic environments delay the healing of bone tissue and produce a relatively insufficient mass of new bone, while also affecting the osseointegration efficiency of the implant (Shan et al., 2017; Uhl, 2018). In contrast, hypoxic conditions promote osteogenic differentiation in vitro. This difference may be the result of a complex in vivo environment. For example, in addition to hypoxic conditions, low-pressure conditions also play an important role in osteogenesis in vivo (Wang et al., 2006; Torigoe et al., 2007). In fact, a low-pressure system has been shown to facilitate the perfusion of a larger number of mesenchymal stem cells into the porous scaffold, enhancing bone formation within the composites (Torigoe et al., 2007). The difference of in vitro and in vivo condition caused the discrepancy of osteogenesis results under normoxia and hypoxia 
environment. To further assess these effects, our future work will include constructing a low-pressure model and determining how different titanium surfaces affect osseointegration.

Collectively, the findings of this study indicate that rough surfaces more effectively promote bone formation in the plains and highlands compared to smooth surfaces. Moreover, under hypoxic conditions, implants with a rough surface enhanced the efficiency of bone binding at an early stage, that is, both at 2 and 4 weeks. However, no significant difference was detected between the two conditions in implants with a rough surface at 4 weeks, suggesting that implant loading should be performed after 4 weeks in highland environments.

\section{CONCLUSION}

Compared to a smooth surface, a rough surface was more conducive to the proliferation of osteogenesis and implant osseointegration. The early enhanced osteogenic differentiation detected on tibial implants under hypoxic conditions appears to be associated with upregulation of the JAK1/STAT1/HIF- $1 \alpha$ pathway. Meanwhile, long-term observation showed that hypoxia inhibited bone formation and osseointegration.

\section{DATA AVAILABILITY STATEMENT}

The raw data supporting the conclusions of this article will be made available by the authors, without undue reservation.

\section{REFERENCES}

Bayram, C., Demirbilek, M., Caliskan, N., Demirbilek, M. E., and Denkbas, E. B. (2012). Osteoblast activity on anodized titania nanotubes: effect of simulated body fluid soaking time. J. Biomed. Nanotechnol. 8, 482-490. doi: 10.1166/ jbn.2012.1391

Bernardi, S., Re, F., Bosio, K., Dey, K., Almici, C., Malagola, M., et al. (2020). Chitosan-hydrogel polymeric scaffold acts as an independent primary inducer of osteogenic differentiation in human mesenchymal stromal cells. Materials 13:3546. doi: 10.3390/ma13163546

Brunello, G., Panda, S., Schiavon, L., Sivolella, S., Biasetto, L., and Del Fabbro, M. (2020). The impact of bioceramic scaffolds on bone regeneration in preclinical in vivo studies: a systematic review. Mater. Aust. 13:1500. doi: 10.3390/ ma13071500

Chen, Y., Han, P., Vandi, L. J., Dehghan-Manshadi, A., Humphry, J., Kent, D., et al. (2019). A biocompatible thermoset polymer binder for direct ink writing of porous titanium scaffolds for bone tissue engineering. Mater. Sci. Eng. C Mater. Biol. Appl. 95, 160-165. doi: 10.1016/j.msec.2018.10.033

Cohen, D. J., Scott, K. M., Kulkarni, A. N., Wayne, J. S., Boyan, B. D., and Schwartz, Z. (2020). Acellular mineralized allogenic block bone graft does not remodel during the 10 weeks following concurrent implant placement in a rabbit femoral model. Clin. Oral Implants Res. 31, 37-48. doi: 10.1111/ clr. 13544

Corvino, E., Pesce, P., Mura, R., Marcano, E., and Canullo, L. (2020). Influence of modified titanium abutment surface on peri-implant soft tissue behavior: a systematic review of in vitro studies. Int. J. Oral Maxillofac. Implants 35, 503-519. doi: 10.11607/jomi.8110

Dashnyam, K., Buitrago, J. O., Bold, T., Mandakhbayar, N., Perez, R. A., Knowles, J. C., et al. (2019). Angiogenesis- promoted bone repair with silicate-shelled hydrogel fiber scaffolds. Biomater. Sci. 7, 5221-5231. doi: 10.1039/c9bm01103j

\section{ETHICS STATEMENT}

The animal study was reviewed and approved by Ethics Committee of Long Guixingke Animal Farm, Baiyun District, Guangzhou.

\section{AUTHOR CONTRIBUTIONS}

MR, YW, and PC conceived and designed the study. YW, ZG, HL, ZL, PS, JZ, WY, HC, RY, and YY performed the experiments and analyzed the data. YW wrote the manuscript. YW, ZG, PC, and MR revised the manuscript. All authors contributed to the article and approved the submitted version.

\section{FUNDING}

This study was funded by the Natural Science Foundation of Guangdong Province (grant number 2016A030310240), the Guangdong Science and Technology Innovation Strategy Special Fund Project (grant number 2018KJY2014), the Medical Research Foundation of Guangdong Province (grant number B2019117), the Guangdong Provincial Administration of Traditional Chinese Medicine, Chinese Medicine Research Project (grant number 20202129), the Natural Science Foundation of Tibet Autonomous Region (grant number XZ2017ZR-ZYZ37), and the National Natural Science Foundation of China for Youth (grant number 81600900).

Ding, X., Zhou, L., Wang, J., Zhao, Q., Lin, X., Gao, Y., et al. (2015). The effects of hierarchical micro/nanosurfaces decorated with $\mathrm{TiO} 2$ nanotubes on the bioactivity of titanium implants in vitro and in vivo. Int. J. Nanomedicine 10, 6955-6973. doi: 10.2147/IJN.S87347

Du, Y., Ge, Y., Xu, Z., Aa, N., Gu, X., Meng, H., et al. (2018). Hypoxiainducible factor 1 alpha (HIF- 1alpha)/vascular endothelial growth factor (VEGF) pathway participates in angiogenesis of myocardial infarction in Muscone-treated mice: preliminary study. Med. Sci. Monit. 24, 8870-8877. doi: 10.12659/MSM.912051

Feng, H., Xing, W., Han, Y., Sun, J., Kong, M., Gao, B., et al. (2020). Tendonderived cathepsin K-expressing progenitor cells activate hedgehog signaling to drive heterotopic ossification. J. Clin. Invest. 130, 6354-6365. doi: 10.1172/ JCI132518

Gonzaga, M. G., Dos, S. K. B., de Figueiredo, F., Feldman, S., Ervolino, E., Dos Santos, M. C. G., et al. (2019). Effectiveness of rhBMP-2 association to autogenous, allogeneic, and heterologous bone grafts. Microsc. Res. Tech. 82, 689-695. doi: 10.1002/jemt.23215

Guglielmotti, M. B., Olmedo, D. G., and Cabrini, R. L. (2019). Research on implants and osseointegration. Periodontol. 2000, 178-189. doi: 10.1111/ prd.12254

He, W., Yin, X., Xie, L., Liu, Z., Li, J., Zau, S., et al. (2019). Enhancing osseointegration of titanium implants through large-grit sandblasting combined with micro-arc oxidation surface modification. J. Mater. Sci. Mater. Med. 30:73. doi: $10.1007 /$ s10856-019-6276-0

He, W., Zhang, H., and Qiu, J. (2020). Osteogenic effects of bioabsorbable magnesium implant in rat mandibles and in vitro. J. Periodontol. 92, 1181-1191. doi: 10.1002/JPER.20-0162

Horvathy, D. B., Vacz, G., Szabo, T., Szigyarto, I. C., Toro, I., Vámos, B., et al. (2016). Serum albumin coating of demineralized bone matrix results in stronger new bone formation. J. Biomed. Mater. Res. B Appl. Biomater. 104, 126-132. doi: $10.1002 /$ jbm.b.33359 
Kim, S., Choi, J. Y., Jung, S. Y., Kang, H. K., Min, B. M., Yeo, I. L., et al. (2019). A laminin-derived functional peptide, PPFEGCIWN, promotes bone formation on sandblasted, large-grit, acid-etched titanium implant surfaces. Int. J. Oral Maxillofac. Implants 34, 836-844. doi: 10.11607/jomi.7178

Lee, J., and Bance, M. L. (2019). Physiology of osseointegration. Otolaryngol. Clin. N. Am. 52, 231-242. doi: 10.1016/j.otc.2018.11.004

Lee, S. H., Moon, J. H., Jeong, C. M., Bae, E. B., Park, C. E., Jeon, G. R., et al. (2017). The mechanical properties and biometrical effect of 3D preformed titanium membrane for guided bone regeneration on alveolar bone defect. Biomed. Res. Int. 2017:7102123. doi: 10.1155/2017/7102123

Lezon, C., Bozzini, C., Aguero, R. A., Pinto, P., Champin, G., Alippi, R. M., et al. (2016). Effect of chronic undernutrition on body mass and mechanical bone quality under normoxic and altitude hypoxic conditions. Br. J. Nutr. 115, 1687-1695. doi: 10.1017/S000711451600060X

Liu, S., Chow, I., Lu, L., Ren, Y. M., Yang, H. L., Jian, S. Y., et al. (2020a). Comparison of sleep disturbances Between older nursing home residents in high- and low-altitude areas. J. Geriatr. Psychiatry Neurol. 33, 370-376. doi: $10.1177 / 0891988719892335$

Liu, P., Hao, Y., Zhao, Y., Yuan, Z., Ding, Y., and Cai, K. (2017). Surface modification of titanium substrates for enhanced osteogenetic and antibacterial properties. Colloids surf. Colloids Surf. B Biointerfaces 160, 110-116. doi: 10.1016/j.colsurfb.2017.08.044

Liu, Y., Huang, X., Yu, H., Yang, J., Li, Y., Yuan, X., et al. (2019). HIF-1alphaTWIST pathway restrains cyclic mechanical stretch-induced osteogenic differentiation of bone marrow mesenchymal stem cells. Connect. Tissue Res. 60, 544-554. doi: 10.1080/03008207.2019.1601185

Liu, J., Kang, Y., Yin, S., Chen, A., Wu, J., Liang, H., et al. (2020b). Key role of microtubule and its acetylation in a zinc oxide nanoparticle-mediated lysosome-autophagy system. Small 16:e2001857. doi: 10.1002/smll.202001857

Liu, F., Liang, T., Zhang, Z., Liu, L., Li, J., Dong, W., et al. (2021). Effects of altitude on human oral microbes. AMB Express 11:41. doi: 10.1186/ s13568-021-01200-0

Lu, X., Xiong, S., Chen, Y., Zhao, F., Hu, Y., Guo, Y., et al. (2020). Effects of statherin on the biological properties of titanium metals subjected to different surface modification. Colloids Surf. B Biointerfaces 188:110783. doi: 10.1016/j. colsurfb.2020.110783

Ma, D., Zheng, B., Liu, H. L., Zhao, Y. B., Liu, X., Zhang, X. H., et al. (2020). Klf5 down-regulation induces vascular senescence through eIF5a depletion and mitochondrial fission. PLoS Biol. 18:e3000808. doi: 10.1371/journal.pbio.3000808

Mavrogenis, A. F., Dimitriou, R., Parvizi, J., and Babis, G. C. (2009). Biology of implant osseointegration. J. Musculoskelet. Neuronal Interact. 9, 61-71.

Pippenger, B. E., Rottmar, M., Kopf, B. S., Stubinger, S., Dalla, T. F., Berner, S., et al. (2019). Surface modification of ultrafine-grained titanium: influence on mechanical properties, cytocompatibility, and osseointegration potential. Clin. Oral Implants Res. 30, 99-110. doi: 10.1111/clr.13396

Shan, Z., Luo, Z. P., Shen, X., and Chen, L. (2017). Promotion of fracture healing by conjugated linoleic acid in rats. J. Orthop. Surg. 25:613379198. doi: $10.1177 / 2309499017718910$

Suresh, S., Rajvanshi, P. K., and Noguchi, C. T. (2019). The many facets of erythropoietin physiologic and metabolic response. Front. Physiol. 10:1534. doi: $10.3389 /$ fphys.2019.01534

Talley, A. D., Boller, L. A., Kalpakci, K. N., Shimko, D. A., Cochran, D. L., and Guelcher, S. A. (2018). Injectable, compression-resistant polymer/ceramic composite bone grafts promote lateral ridge augmentation without protective mesh in a canine model. Clin. Oral Implants Res. 29, 592-602. doi: 10.1111/ clr. 13257

Tan, J., Liu, Z., Wang, D., Zhang, X., Qian, S., and Liu, X. (2020). A facile and universal strategy to endow implant materials with antibacterial ability via alkalinity disturbing bacterial respiration. Biomater. Sci. 8, 1815-1829. doi: $10.1039 / \mathrm{c} 9 \mathrm{bm} 01793 \mathrm{c}$

Torigoe, I., Sotome, S., Tsuchiya, A., Yoshii, T., Takahashi, M., Kawabata, S., et al. (2007). Novel cell seeding system into a porous scaffold using a modified low-pressure method to enhance cell seeding efficiency and bone formation. Cell Transplant. 16, 729-739. doi: 10.3727/000000007783465109

Uhl, E. W. (2018). The pathology of vitamin D deficiency in domesticated animals: An evolutionary and comparative overview. Int. J. Paleopathol. 23, 100-109. doi: 10.1016/j.ijpp.2018.03.001

Wang, J., Asou, Y., Sekiya, I., Sotome, S., Orii, H., and Shinomiya, K. (2006). Enhancement of tissue engineered bone formation by a low pressure system improving cell seeding and medium perfusion into a porous scaffold. Biomaterials 27, 2738-2746. doi: 10.1016/j.biomaterials.2005.12.005

Wen, D. D., and Lv, Y. L. (2020). Effect of different doses of aspirin on the early osseointegration of titanium alloy implants in rats. J. Dent. Prevent Treat. 28, 285-291. doi: 10.12016/j.issn.2096-1456.2020.05.003

Wu, J., Lai, X., Cui, G., Chen, Q., Liu, J., Kang, Y., et al. (2020a). Dual effects of JNK activation in blood-milk barrier damage induced by zinc oxide nanoparticles. J. Hazard. Mater. 399:122809. doi: 10.1016/j. jhazmat.2020.122809

Wu, T., Li, B., Wang, W., Chen, L., Li, Z., Wang, M., et al. (2020b). Strontiumsubstituted hydroxyapatite grown on graphene oxide nanosheet-reinforced chitosan scaffold to promote bone regeneration. Biomater. Sci. 8, 4603-4615. doi: $10.1039 / \mathrm{d} 0 \mathrm{bm} 00523 \mathrm{a}$

Wu, X., Liu, S., Hu, Z., Zhu, G., Zheng, G., and Wang, G. (2018a). Enriched housing promotes post-stroke neurogenesis through calpain 1-STAT3/ HIF-1alpha/VEGF signaling. Brain Res. Bull. 139, 133-143. doi: 10.1016/j. brainresbull.2018.02.018

Wu, S., Zhang, A., Li, S., Chatterjee, S., Qi, R., Segura-Ibarra, V., et al. (2018b). Polymer functionalization of isolated mitochondria for cellular transplantation and metabolic phenotype alteration. Adv. Sci. 5:1700530. doi: 10.1002/ advs. 201700530

Xue, Y., Li, Z., Wang, Y., Zhu, X., Hu, R., and Xu, W. (2020). Role of the HIFlalpha/SDF1/CXCR4 signaling axis in accelerated fracture healing after craniocerebral injury. Mol. Med. Rep. 22, 2767-2774. doi: 10.3892/ mmr.2020.11361

Yang, M., Wang, L., Wang, X., Wang, X., Yang, Z., Li, J., et al. (2017). IL-6 promotes FSH-induced VEGF expression through JAK/STAT3 signaling pathway in bovine granulosa cells. Cell. Physiol. Biochem. 44, 293-302. doi: $10.1159 / 000484885$

Yu, Y., Ma, L., Zhang, H., Sun, W., Zheng, L., Liu, C., et al. (2020). EPO could be regulated by HIF-1 and promote osteogenesis and accelerate bone repair. Artif. Cells Nanomed. Biotechnol. 48, 206-217. doi: 10.1080/21691401.2019.1699827

Zhang, L., Wang, Y., Ma, J., Lai, X., Fang, J., Li, G., et al. (2017). Exogenous MSCs ameliorate hypoxia/reoxygenation injury in renal tubular epithelial cells through JAK/STAT signaling pathway- mediated regulation of HMGB1. Am. J. Transl. Res. 9, 2412-2420.

Conflict of Interest: The authors declare that the research was conducted in the absence of any commercial or financial relationships that could be construed as a potential conflict of interest.

Publisher's Note: All claims expressed in this article are solely those of the authors and do not necessarily represent those of their affiliated organizations, or those of the publisher, the editors and the reviewers. Any product that may be evaluated in this article, or claim that may be made by its manufacturer, is not guaranteed or endorsed by the publisher.

Copyright (๑) 2021 Wang, Gan, Lu, Liu, Shang, Zhang, Yin, Chu, Yuan, Ye, Chen and Rong. This is an open-access article distributed under the terms of the Creative Commons Attribution License (CC BY). The use, distribution or reproduction in other forums is permitted, provided the original author(s) and the copyright owner(s) are credited and that the original publication in this journal is cited, in accordance with accepted academic practice. No use, distribution or reproduction is permitted which does not comply with these terms. 